\title{
Proposal and Analysis of a Hybrid Silicon Photonic-Lithium Niobate Waveguide for Difference Frequency Generation
}

\author{
Marc Savanier $^{\mathrm{a}}$, Peter O. Weigel ${ }^{\mathrm{a}}$, Shayan Mookherjea ${ }^{\mathrm{a}, *}$ \\ ${ }^{a}$ Department of Electrical and Computer Engineering, University of California, San Diego, \\ 9500 Gilman Drive, Mail Code 0407, La Jolla, California 92093-0407, USA
}

\begin{abstract}
Integrated optics devices for nonlinear optics may be made by using unpatterned thin films of a nonlinear crystal such as lithium niobate in conjunction with (for example, bonded to) an easily-patterned material such as silicon or silicon nitride which is commonly used in a silicon photonics platform. We propose and analyze a device for difference-frequency generation in a hybrid waveguide which uses the strongest nonlinear tensor coefficient without ion-exchanging, etching, or periodically-poling lithium niobate, which can considerably simplify the fabrication process.
\end{abstract}

Keywords: Nonlinear optics, Optical Waveguides, Silicon Photonics,

Wavelength conversion

2010 MSC: 78A60, 78A50

\section{Introduction}

The conventional fabrication techniques of periodically-poled crystals for nonlinear optics [1] are quite different from the fabrication methods used to make modern micro-electronics integrated circuits and silicon photonics. Based on this observation, the intention of this paper is to describe a hybrid silicon photonics waveguide for optical wavelength conversion. Here, we propose and

\footnotetext{
${ }^{*}$ Corresponding author

Email address: smookherjea@eng.ucsd.edu (Shayan Mookherjea)
}

Preprint submitted to Optics Communications

August 3, 2015

(C) 2015. This manuscript version is made available under the Elsevier user license http://www.elsevier.com/open-access/userlicense/1.0/ 
study a simple way of incorporating the nonlinearity of lithium niobate (LN), the workhorse material for nonlinear optics, and similar materials in a silicon photonics device, without periodically poling or etching the crystal, and relying only on bonding 2 .

As shown in Fig. 1, our proposed waveguide consists of an unpatterned thin film of LN bonded (or otherwise placed in proximity to) to a thin film of a dielectric material (e.g., silicon nitride, SiN), semiconductor (e.g., crystalline or amorphous silicon, Si) or polymer [3, 4, 5, 6, 7, 8, This second film is patterned before bonding to create a rib-loaded waveguide in the transverse cross-section, and a periodic pattern along the direction of propagation. This is a standard lithography procedure in silicon photonics. Thin-film LN on low-refractive-index handle (e.g., $\mathrm{SiO}_{2}$ ) is available commercially (e.g., NanoLN or SRICO, Inc.). It may be simply left as an unpatterned slab. The optical mode is thus a hybrid one, partially distributed in the LN slab, and partially in the second material.

The main advantages of this new device arise from taking advantage of modern CMOS-compatible silicon photonics fabrication methods to simplify the processing of $\mathrm{LN}$ :

1. This geometry allows us to use the lowest-order (quasi)-TE polarization modes in each of the waves, which is preferred for planar integrated optics, and the highest-magnitude nonlinear coefficient $\left(d_{33}\right)$ of LN in the convenient $x$-cut orientation. In contrast, challenges with periodically poling $\mathrm{x}$-cut LN have usually led researchers to require z-cut LN, thus forcing the use of TM-polarized waves, which are not the lowest-order modes in silicon photonic waveguides, and suffer from higher propagation and bending losses 9 .

2. The LN film is not etched; instead, a transverse mode is defined by the rib of the second material, made of $\mathrm{Si}, \mathrm{SiN}$, or some material, which is easier to pattern, and in which high-quality and complex waveguiding structures have already been shown [9, 10. In contrast, conventional approaches have attempted etching, ion-milling or sawing LN, which can increase the 
propagation loss or impact the nonlinear coefficient [11, 12].

3. LN does not have to be periodically poled. As an alternative to the conventional technique of periodic poling, one may use the technique of "balanced phase matching" (BPM) whereby the second of each pair of segments along the direction of propagation of the hybrid mode is used to periodically compensate for the phase mismatch incurred in nonlinear interactions in the first segment 11, 13, 14. Although periodic poling will, in general, result in a higher conversion efficiency, the simple approach for implementing BPM in this hybrid silicon photonics platform may be attractive for many cost-sensitive applications.

\section{Nonlinear optics: difference frequency generation}

Some crystals such as LN have a strong second-order optical nonlinearity based on the $\chi^{(2)}$ tensor, and are widely used for optical wavelength conversion, in which one or more optical waves (or spectral components of a pulse or a frequency comb) mix and generate new spectral tones at the sum and/or difference frequencies [1. This process is governed by the principles of energy conservation and phase-matching.

Focusing here on the case of difference frequency generation (DFG) in order to be specific, if we label the input optical radial frequencies as $\omega_{2}$ and $\omega_{3}$ (assuming $\omega_{3}<\omega_{2}$ ), then the newly generated frequency is at $\omega_{1}=\omega_{2}-\omega_{3}$, and it is required that the wave vectors satisfy the phase-matching relationship $\mathbf{k}\left(\omega_{2}\right)-\mathbf{k}\left(\omega_{3}\right)-\mathbf{k}\left(\omega_{1}\right)=0$. The most convenient technique of phase-matching requires "assisting" the wave vector sum by some induced technique, e.g., using lithography and poling to periodically reverse the sign of the nonlinear coefficient along the waveguide [see Fig. 1(a)], which is known as quasi-phase matching (QPM) 15.

\subsection{Formulation}

To study the device in detail, we write the nonlinear Helmholtz equation for the field evolution of a field $\mathbf{E}_{1}$ generated by DFG from undepleted pump fields 
$\mathbf{E}_{2}$ and $\mathbf{E}_{3}$,

$$
\nabla^{2}\left(\mathbf{E}_{1}\right)_{i}+\frac{\omega_{1}^{2}}{c^{2}} \epsilon_{\mathrm{rel}}(\mathbf{r})\left(\mathbf{E}_{1}\right)_{i}+\frac{\omega_{1}^{2}}{c^{2} \epsilon_{0}} 2 \epsilon_{0} \chi_{i j k}^{(2)}\left(\mathbf{E}_{2}\right)_{j}\left(\mathbf{E}_{3}^{*}\right)_{k}=0
$$

where $\epsilon_{\text {rel }}(\mathbf{r})$ describes the pattern in the (relative) dielectric permittivity shown in Fig. 1, and the subscripts in $\chi_{i j k}^{(2)}$ and the fields describe which tensor coefficient is involved, based on the 'i', 'j' and ' $\mathrm{k}$ ' polarizations of $\mathbf{E}_{1}, \mathbf{E}_{2}$ and $\mathbf{E}_{3}$, respectively.

Based on the dielectric distribution shown in Fig. 1 (b), we assume a solution of the form:

$$
\mathbf{E}_{1}=\left\{\begin{array}{c}
A_{1}(z) \mathbf{E}_{1}^{A}\left(\mathbf{r}_{\perp}\right) \exp \left(i \beta_{1}^{A} z\right)+\text { c.c. } \\
\text { for } 0<z-m \Lambda \leq \Lambda^{A} \\
B_{1}(z) \mathbf{E}_{1}^{B}\left(\mathbf{r}_{\perp}\right) \exp \left(i \beta_{1}^{B} z\right)+\text { c.c. }, \\
\text { for } \Lambda^{A}<z-m \Lambda \leq \Lambda^{A}+\Lambda^{B}
\end{array}\right.
$$

where $\Lambda=\Lambda^{A}+\Lambda^{B}$ is the periodicity, and $m$ is an integer. Here, we are describing devices in which $\Lambda^{A, B}$ are far from Bragg resonance, and strong coupling to counter-propagating modes can be ignored. The amplitude coefficients $A_{1}$ and $B_{1}$ are linked by field continuity conditions at the interfaces between the segments. We have assumed a normalization in which the optical power of the field is defined in terms of the amplitude by the relationship $P=2 n \epsilon_{0} c|A|^{2}$, with $n$ the mode effective index.

Substituting Eq. (2) in Eq. (1), and under the usual slowly-varying envelope approximation, we get

$$
\begin{aligned}
\frac{d A_{1}}{d z}= & +i \frac{2 \omega_{1}^{2}}{c^{2} \beta_{1}^{A}} A_{2} A_{3}^{*} \overbrace{\int d \mathbf{r}_{\perp} \frac{1}{2} \chi_{i j k}^{(2)}\left(\mathbf{E}_{1}^{A}\right)_{i}\left(\mathbf{E}_{2}^{A}\right)_{j}\left(\mathbf{E}_{3}^{A}\right)_{k}}^{\Gamma_{i j k}^{A}} \\
& \times \exp [i \underbrace{\left.\beta_{2}^{A}-\beta_{3}^{A}-\beta_{1}^{A}\right)}_{\Delta \beta^{A}} z]
\end{aligned}
$$

and a similar equation for segment $\mathrm{B}$, obtained by substituting the letter ' $\mathrm{A}$ ' by 'B' everywhere in Eq. (3). The second-order nonlinear coefficient $(\Gamma)$ and phase-mismatch $(\Delta \beta)$ are defined as indicated, and may take different values in segments A and B. 
In the following discussion, we choose the fields to be TE-polarized waveguide modes polarized along the crystal axis [see Fig. 1(b)] so that we may use the (Kleinman contracted notation) $\Gamma_{33}$ coefficient which is the largest-magnitude second-order nonlinear tensor coefficient in LN. In this case, $\Delta \beta$ is not zero and the governing equations are

$$
\begin{array}{ll}
\frac{d A_{1}}{d z}=\frac{2 i \omega_{1}^{2}}{c^{2} \beta_{1}^{A}} \Gamma^{A} A_{2} A_{3}^{*} e^{i \Delta \beta^{A} z}, & \text { for } 0<z-m \Lambda \leq \Lambda^{A}, \\
\frac{d B_{1}}{d z}=\frac{2 i \omega_{1}^{2}}{c^{2} \beta_{1}^{B}} \Gamma^{B} B_{2} B_{3}^{*} e^{i \Delta \beta^{B} z}, & \text { for } \Lambda^{A}<z-m \Lambda \leq \Lambda .
\end{array}
$$

\subsection{Numerical study}

To demonstrate how the field evolves, in Fig. 3(a) we show a numerical calculation of the growth of a mid-infrared (mid-IR, wavelength $\lambda_{1}=3.255 \mu \mathrm{m}$ ) field generated by DFG from near-infrared (NIR, wavelengths $\lambda_{2}=1.05 \mu \mathrm{m}$ and $\left.\lambda_{3}=1.55 \mu \mathrm{m}\right)$ pump fields.

As shown in Fig. 1(b), we assume a device that consists of an x-cut LNon- $\mathrm{SiO}_{2}$ slab, bonded to a lithographically patterned film of $\mathrm{SiN}$ on low-index substrate (e.g., $\mathrm{SiO}_{2}$ to match the upper and lower cladding indices of the hybrid waveguide). The hybrid waveguide is thus formed: an unpatterned LN slab loaded with a SiN rib waveguide. LN provides the $\chi^{(2)}$ nonlinearity for DFG; SiN does not have a significant nonlinearity, and instead helps define the mode and the two-section pattern.

For simulation purposes, we assume the thin-film LN thickness is $0.5 \mu \mathrm{m}$. Unlike the sketch of Fig. 1.(b), where the SiN waveguide height is kept constant and its width is varied, we assume here the width of the $\mathrm{SiN}$ waveguide is $3.0 \mu \mathrm{m}$, and its surface is periodically corrugated instead. Its height alternatively varies between $0.20 \mu \mathrm{m}$ (Section A) and $0.25 \mu \mathrm{m}$ (Section B). Such a structure may be harder to fabricate experimentally than one in which the width is varied, but shows the same waveguiding principles from a theoretical perspective. Also, while the experimentally fabricated structure will have smooth transitions between the sections, here we assume that the waveguide cross-section in each 
section is constant, in order to make the correspondence between theory and numerical simulations easier.

Modes were calculated using a vectorial finite-difference eigenmode solver (Lumerical), and the field evolution of the DFG amplitude was obtained by direct numerical integration of Eq. (4). For each cross-section of the device, the profiles of the TE-polarized modes involved in the DFG process are given in Fig. 2. We can see that the interacting fundamental modes are similar, with comparable effective areas, despite their dissimilar wavelengths. Comparing the hybrid modes in both sections, we see that the fraction of the field in the nonlinear LN layer is higher in Section A (the modes are 'pulled' in the LN layer) than in Section B (the modes are 'pushed' in the SiN waveguide), resulting in $\Gamma^{A}>\Gamma^{B}$. The lengths $\Lambda^{A}$ and $\Lambda^{B}$ for this structure were chosen to be $3.36 \mu \mathrm{m}$ and $3.19 \mu \mathrm{m}$, respectively. As detailed in the next subsection, they correspond to the coherence length $\Lambda=\pi / \Delta \beta$ of each section.

The result of the simulations is that we observe, as shown in Fig. 3, the net mid-IR field grows quadratically with distance (i.e., the usual favorable behavior of the DFG field), and the device is capable of generating more than $100 \mu \mathrm{W}$ mid-IR power in less than $4 \mathrm{~mm}$ using $100 \mathrm{~mW}$ NIR pumps. To explain the reason for this cumulative growth of the DFG field, even though no periodic poling was assumed in the LN slab, we turn to an analytical formalism which dates back about 25 years.

\subsection{Analytical study}

The method for theoretical analysis of Eq. (4) is based on a technique called "balanced phase matching" [13, 14. For analytical convenience, we assume that the waveguide cross-sections are uniform in each of sections $\mathrm{A}$ and $\mathrm{B}$, with the only change occurring at the interfaces. Then, Eq. (4) can be integrated with regard to the shifted coordinate $z^{\prime}=z-m \Lambda-\Lambda^{A}$,

$$
B_{1}\left(\Lambda^{B}\right)=B_{1}(0)+\frac{2 i \omega_{1}^{2}}{c^{2} \beta_{1}^{B}} \Gamma^{B} B_{2} B_{3}^{*} \frac{e^{i \Delta \beta^{B} \Lambda^{B}}-1}{i \Delta \beta^{B}}
$$


where

$$
B_{1}(0)=\frac{2 i \omega_{1}^{2}}{c^{2} \beta_{1}^{A}} \Gamma^{A} A_{2} A_{3}^{*} \frac{e^{i \Delta \beta^{A} \Lambda^{A}}-1}{i \Delta \beta^{A}} e^{i \beta_{1}^{A} \Lambda^{A}} .
$$

After propagating through Section A and B, the DFG field at the start of the next section A has the amplitude

$$
A_{1}(0) \equiv B_{1}\left(\Lambda^{B}\right) e^{i \beta_{1}^{B} \Lambda^{B}}
$$

where $B_{1}$ is given by Eq. 5a , and whose further evolution is driven by a nonlinear polarization that is proportional to

$$
A_{2} e^{i\left(\beta_{2}^{A} \Lambda^{A}+\beta_{2}^{B} \Lambda^{B}\right)} A_{3}^{*} e^{-i\left(\beta_{3}^{A} \Lambda^{A}+\beta_{3}^{B} \Lambda^{B}\right)} .
$$

For the DFG field to grow cumulatively after each section A and B, we select the section length $\Lambda^{B}$ so that Eq. 6a has the same phase relationship to Eq. 6b. as $B_{1}(0)$ did to $B_{2} B_{3}^{*}=A_{2} e^{i \beta_{2}^{A} \Lambda^{A}} A_{3}^{*} e^{-i \beta_{3}^{A} \Lambda^{A}}$.

Because $\Delta \beta^{A} \neq 0$ here, we choose the section length $\Lambda^{A}$ such that $\Delta \beta^{A} \Lambda^{A}=$ $\pi$, which maximizes the DFG field growth in the first section. With this substitution, Eq. 6a becomes

$$
\begin{aligned}
A_{1}(0)= & \frac{4 \omega_{1}^{2}}{c^{2}} A_{2} A_{3}^{*} e^{i\left(\beta_{1}^{A} \Lambda^{A}+\beta_{1}^{B} \Lambda^{B}\right)} \\
& \times \frac{\Gamma^{A}}{\Delta \beta^{A} \beta_{1}^{A}}\left[1+\frac{\eta}{2}\left(e^{i \Delta \beta^{B} \Lambda^{B}}-1\right)\right]
\end{aligned}
$$

where

$$
\eta=\frac{\Gamma^{B}}{\Delta \beta^{B} \beta_{1}^{B}} / \frac{\Gamma^{A}}{\Delta \beta^{A} \beta_{1}^{A}} .
$$

The term in square brackets on the second line of Eq. $7 \mathrm{~b}$ is complex, and we need to select the length of Section B such that the field components have the correct phase relationship at the end of the segment (as stated in the text following Eq 6b. Thus, we choose the section length $\Lambda^{B}$ such that

$$
\text { phase }\left[1+\frac{\eta}{2}\left(e^{i \Delta \beta^{B} \Lambda^{B}}-1\right)\right]=\Delta \beta^{B} \Lambda^{B}+(2 p+1) \pi
$$

where $p$ is any integer. A solution of this transcendental equation is $\Lambda^{B}=$ $\pi / \Delta \beta^{B}$. Integer multiples of $2 \pi$ may be added to the right-hand side of this 
equation: although the device length increases, the propagation loss of a lowduty-cycle periodic structure may be lower [16]. We require $\eta \neq 1$ so that the amplitude decay in Section B does not cancel the growth in Section A. Note that $\eta=-1$ is a special case which corresponds to traditional QPM. The case $\eta=0$ means that is no nonlinear generation in Section B, and that section only serves to re-phase the interacting waves for the next Section A that follows it.

From Eq. $7 \mathrm{~b}$, the output power $\left(P_{1}\right)$ after $N$ segments (each consisting of a Section A and a Section B) driven by input powers $P_{2}$ and $P_{3}$ is

$$
P_{1}=\underbrace{\frac{8 \pi^{2} \Gamma_{\mathrm{eff}}^{2}}{n_{1} n_{2} n_{3} c \epsilon_{0} \lambda_{1}^{2}}}_{\eta^{0}} L^{2} P_{2} P_{3}
$$

where $L=N \Lambda, \eta^{0}$ is the effective normalized conversion efficiency, and

$$
\Gamma_{\mathrm{eff}}=\Gamma^{A} \frac{2(1-\eta)}{\pi}\left(1+\frac{\left|\Delta \beta^{A}\right|}{\left|\Delta \beta^{B}\right|}\right)^{-1} .
$$

As shown in Fig. 3 by the dashed red line, this theoretical approximation also shows that the net mid-IR field grows quadratically with distance, generating more than $100 \mu \mathrm{W}$ mid-IR power in less than $4 \mathrm{~mm}$ using $100 \mathrm{~mW}$ NIR pumps. Although balanced phase-matching schemes are, in general, less efficient than perfect phase matching or QPM, the modal cross-section area of a hybrid waveguide such as described here (comprised between $1.4 \mu \mathrm{m}^{2}$ and $4.2 \mu \mathrm{m}^{2}$, as shown in Fig. 22 can be smaller than conventional ion-exchanged waveguides, thus boosting the nonlinear conversion efficiency.

\section{Discussion and Conclusion}

For the simulated device structure, the modulation of the nonlinearity $\Gamma$ by $18 \%(\eta=0.721)$ yields $\eta^{0} \sim 7 \% \mathrm{~W}^{-1} \mathrm{~cm}^{-2}$, which is quite impressive for a structure without periodic poling, and without extensive optimization of the device cross-section. Other fabrication approaches avoiding periodic poling, and instead relying on laser lithography, have achieved much smaller conversion efficiencies of $0.8 \times 10^{-6} \% \mathrm{~W}^{-1} \mathrm{~cm}^{-2}$ [17]. In fact, the calculated conversion efficiencies of this structure are higher than those of laser-written waveguides formed 
in periodically-poled $\mathrm{LN}, 3 \times 10^{-4} \% \mathrm{~W}^{-1} \mathrm{~cm}^{-2}$ [18] and $6.5 \% \mathrm{~W}^{-1} \mathrm{~cm}^{-2}$ [19]. Therefore, the bonded hybrid waveguide approach may be a satisfactory way to achieve useful levels of conversion without using periodic poling.

However, it should also be recognized that if periodic poling is acceptable, then the achievable conversion efficiency is at least a factor of two higher (and usually, more than that), since $\eta$ in Eq. 10 can be made negative using QPM, whereas it is always non-negative in the proposed scheme without periodic poling. As stated earlier, the value of this proposed device lies in simplifying the fabrication procedure by requiring only a single uniform 'slab' of lithium niobate which does not have to be periodically poled. The tradeoff is the reduction in the conversion efficiency compared to a periodically-poled structure of similar modal cross-sectional area, which does not require the hybrid waveguide configuration.

Compared to Refs. [13, 14, the main difference in the proposed device is the use of a hybrid waveguide mode consisting of the combination of lithium niobate and silicon or silicon nitride, with patterning implemented only in the silicon or silicon nitride layer. Unlike periodic-poling, features with deep submicron resolution can be patterned easily and cost-effectively at wafer-scale in both silicon and silicon nitride, before dicing into smaller pieces and bonding to lithium niobate. The relatively high index of silicon results in small-area hybrid modes, which can increase the nonlinear conversion efficiency compared to traditional large-area modes; however, the two-photon absorption and free-carrier absorption of silicon at near-infrared wavelengths will also have to be monitored in a practical device. Once the cross-sectional mode profile is calculated (e.g., using a finite-element software package), the analysis of balanced-phase matching proceeds along previously established lines, and the conclusions regarding the periodic growth and decay of the fields are similar as made earlier.

Fig. 4 shows the typical 'sinc-squared'-shaped converted output power versus mid-IR DFG wavelength for $N=600$ segments, while $\lambda_{2}$ is kept constant at 1050 $\mathrm{nm}$ and $\lambda_{3}$ is swept from $1525 \mathrm{~nm}$ to $1575 \mathrm{~nm}$, under the reasonable assumption that $\Gamma^{A}$ and $\Gamma^{B}$ are constant. The full-width at half-maximum bandwidth 
for this structure is about $50 \mathrm{~nm}$. Such spectral acceptance can benefit the tunability of the device, by conveniently mapping the wide tunability of any conventional laser diode at telecom wavelength across the C- and L-bands to the mid-IR range. Further on, like traditional QPM structures, a range of $\Lambda^{A}$ and $\Lambda^{B}$ values - either in a period-chirped structure or in separate structures - are required in order to simultaneously cover a wider mid-IR spectrum. Calculations show that, by varying $\lambda_{2}$ from $1030 \mathrm{~nm}$ to $1080 \mathrm{~nm}$, and accordingly $\lambda_{3}$ from $1450 \mathrm{~nm}$ to $1700 \mathrm{~nm}$, this structure can be used to generate mid-IR wavelengths from about $2.9 \mu \mathrm{m}$ to $3.6 \mu \mathrm{m}$ if an $\mathrm{SiO}_{2}$ cladding is used, as assumed here. Using different cladding materials with infrared transparency may yield a wider tuning range.

Another advantage of using silicon photonics to fabricate the guiding rib structures is that fairly complex circuits may be fabricated on a chip, e.g., waveguide splitters and combiners realized using lithography in the $\mathrm{SiN}$ or $\mathrm{Si}$ layer alone.

Moreover, the two input pump wavelengths considered in this analysis may come from discrete lasers, or spectral lines of an input NIR frequency comb. In such cases, the resulting inherent phase-stability between different nonlinear mixers will be very difficult to achieve otherwise, using separate devices on a table-top.

In these calculations, we have assumed that despite the non-unity value of $\eta$, there is no back-reflection at the interfaces between Sections A and B. From the different mode profiles shown in Fig. 2, it is clear that a practical implementation will require minimal loss adiabatic transitions. One possible solution is to use apodized transitions e.g., waveguide tapers (conveniently fabricated in Section B alone). In this case, the condition $\Delta \beta^{B} \Lambda^{B}=(2 p+1) \pi$ still holds (where $p$ is any integer, whose value can be chosen large enough to accommodate the tapers). In this case, there is no closed-form analytical theory and the performance will have to be optimized using numerical calculations.

In future work, the principal theoretical challenge is to search exhaustively for various hybrid cross-sections which increase the value of $(1-\eta)$ while keeping 
the mode-mismatch propagation losses low at the interfaces between Sections A and B. There is reason for optimism because earlier research suggests that, excluding cases in which a stop-band is formed by the periodicity (which is not the case in the simulations performed here), such losses can be low [20]. The principal experimental challenge is to minimize propagation losses, which like in all guided-wave nonlinear optical structures, can reduce the output power significantly. Regarding the choice of materials, silicon nitride may be a better material for the proposed device due to its lack of significant two photon absorption at near-IR wavelengths (unlike silicon); however, silicon is a good choice for generating mid-IR wavelengths from about $2.9 \mu \mathrm{m}$ to $3.6 \mu \mathrm{m}$.

In summary, we have proposed and analyzed a device for nonlinear interactions between waves which can achieve phase matching without etching, ionexchanging, or periodically poling crystals such as lithium niobate, as long as they can be formed into a hybrid waveguide. This conveniently-manufacturable and compact chip-scale structure has the potential to benefit current research in mid-infrared spectroscopy, free-space communications, laser ranging, imaging, illumination and sensors.

\section{Acknowledgements}

The authors are grateful to the NSF for support (ECCS 1201308) and the Qualcomm Institute CSRO program, S. Sriram and V. E. Stenger (SRICO Inc.) for useful discussions, and A. Lentine, C. DeRose and A. Pomerene (Sandia National Laboratories) for collaboration on silicon photonics.

\section{References}

[1] T. Suhara and M. Fujimura, Waveguide Nonlinear-Optic Devices (Springer, New York, 2003).

[2] S. Mookherjea and M. Savanier, "Heterogeneous Waveguide and Method of Manufacture" US Provisional Patent Application 62/114,884 (2015). 
[3] M. Levy, R. M. Osgood, R. Liu, L. Cross, I. Cargill, A. Kumar, and H. Bakhru, "Fabrication of single- crystal lithium niobate films by crystal ion slicing" Appl. Phys. Lett. 73, 2293-2295 (1998).

[4] P. Rabiei and P. Gunter, "Optical and electro-optical properties of submicrometer lithium niobate slab waveguides prepared by crystal ion slicing and wafer bonding" Appl. Phys. Lett. 85, 4603 (2004).

[5] Y. B. Park, B. Min, K. J. Vahala, and H. A. Atwater, "Integration of singlecrystal LiNbO3 thin film on silicon by laser irradiation and ion implantationinduced layer transfer" Adv. Mater. 18, 1533-1536 (2006).

[6] S. Sriram, V. Stenger, J. Toney, N. Smith, A. Pollick, and J. Scholl. "Electrooptic Devices in Thin Film Lithium Niobate (TFLN ${ }^{\mathrm{TM}}$ )" Proc. Conf. IEEE Avionics, Fiber-Optics and Photonics Technology (AVFOP), TuA3 (2012).

[7] L. Chen, Q. Xu, M. G. Wood, and R. M. Reano, "Hybrid silicon and lithium niobate electro-optical ring modulator" Optica 1, 112-118 (2014).

[8] L. Cao, A. Aboketaf, Z. Wang, and S. Preble, "Hybrid amorphous silicon (aSi:H)-LiNbO3 electro-optic modulator" Opt. Commun. 330, 40-44 (2014).

[9] Y. A. Vlasov and S. McNab, "Losses in single-mode silicon-on-insulator strip waveguides and bends," Opt. Express 12, 1622-1631 (2004).

[10] S. Ates, I. Agha, M. Davanco, and K. Srinivasan, "Frequency conversion interface to the telecom band via four-wave mixing Bragg scattering in a silicon nitride chip," in Frontiers in Optics 2013, P. Delyett, Jr. and D. Gauthier, eds., OSA Technical Digest (online) (Optical Society of America, 2013), paper LW1G.4.

[11] C. Restoin, S. Massy, C. Darraud-Taupiac, A. Barthelemy, "Fabrication of $1 \mathrm{D}$ and 2D structures at submicrometer scale on lithium niobate by electron beam bombardment" Optical Materials, 22 193-199 (2003). 
[12] F. Lacour, N. Courjal, M.-P. Bernal, A. Sabac, C. Bainier, M. Spajer, "Nanostructuring lithium niobate substrates by focused ion beam milling" Optical Materials, 27, 1421-1425 (2005).

[13] J. Bierlein, D. Laubacher, J. Brown, and C. Van der Poel, "Balanced phase matching in segmented KTiOPO4 waveguides" Appl. Phys. Lett. 56, 17251727 (1990).

[14] J. Khurgin, S. Colak, R. Stolzenberger, and R. N. Bhargava, "Mechanism for efficient blue second-harmonic generation in periodically segmented waveguides" Appl. Phys. Lett. 57, 2540-2542 (1990).

[15] M. M. Fejer, G. Magel, D. H. Jundt, and R. L. Byer, "Quasi-phase-matched second harmonic generation: tuning and tolerances" IEEE J. Quantum Electron. 28, 2631-2654 (1992).

[16] D. D. Stancil, "Kronig-Penney model for periodically segmented waveguides" Appl. Opt. 35, 4767 (1996).

[17] J. Thomas, V. Hilbert, R. Geiss, T. Pertsch, A. Tunnermann and S. Nolte, "Quasi phase matching in femtosecond pulse volume structured x-cut lithium niobate". Laser \& Photonics Rev. 7(3), L17-L20 (2013)

[18] Y. L. Lee, N. E. Yu, C. Jung, B. A. Yu, I. B. Sohn, S. C. Choi, Y. C. Noh, D. K. Ko, W. S. Yang, H. M. Lee, W. K. Kim, and H. Y. Lee, "Second-harmonic generation in periodically poled lithium niobate waveguides fabricated by femtosecond laser pulses" Appl. Phys. Lett. 89, 171103 (2006).

[19] R. Osellame, M. Lobino, N. Chiodo, M. Marangoni, G. Cerullo, R. Ramponi, H. T. Bookey, R. R. Thomson, N. D. Psaila, and A. K. Kar, "Femtosecond laser writing of waveguides in periodically poled lithium niobate preserving the nonlinear coefficient" Appl. Phys. Lett. 90, 1107 (2007).

[20] L. Li and J. J. Burke, "Linear propagation characteristics of periodically segmented waveguides" Opt. Lett. 17, 1195-1197 (1992). 

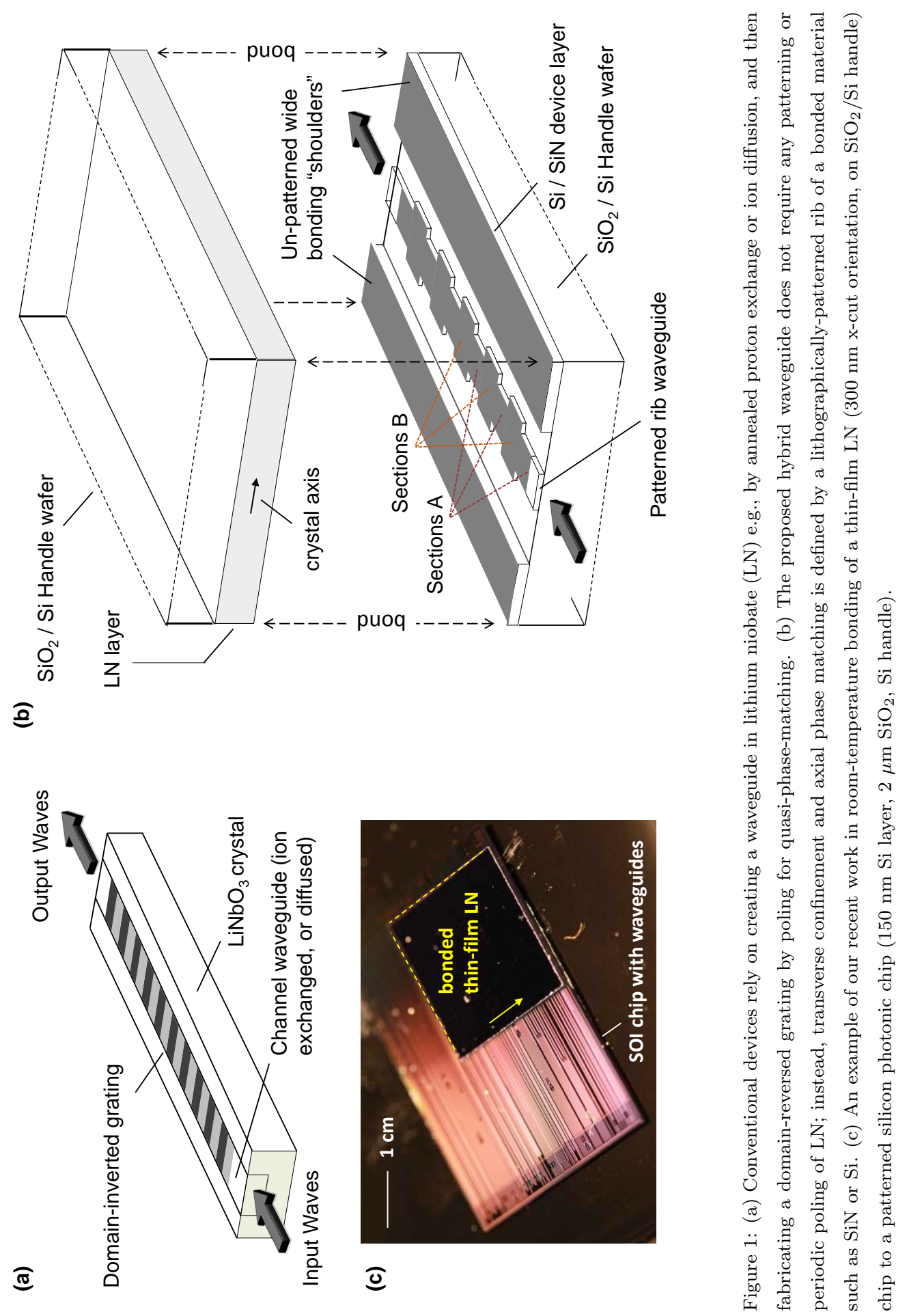


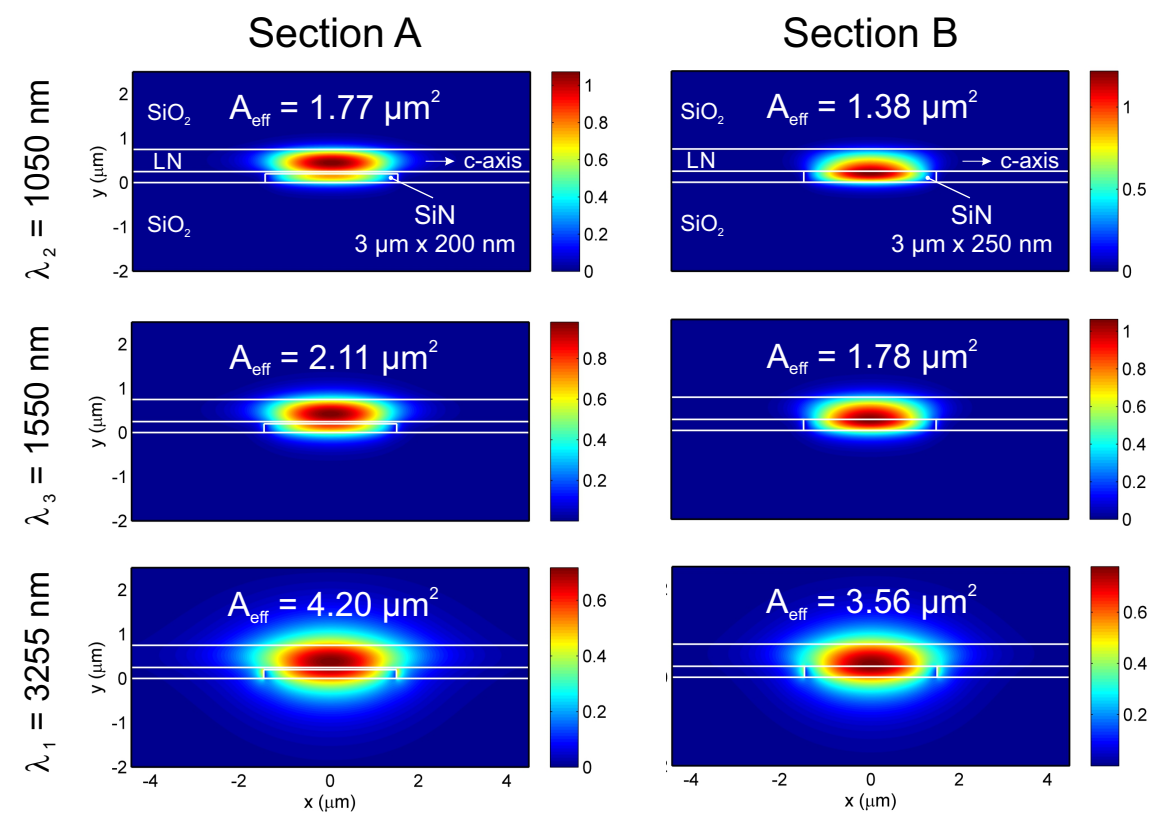

Figure 2: Hybrid waveguide cross-section and simulated $\mathrm{TE}_{00}$ mode profiles (normalized to unity power), in Section A and Section B, at the wavelengths involved in the DFG process. 

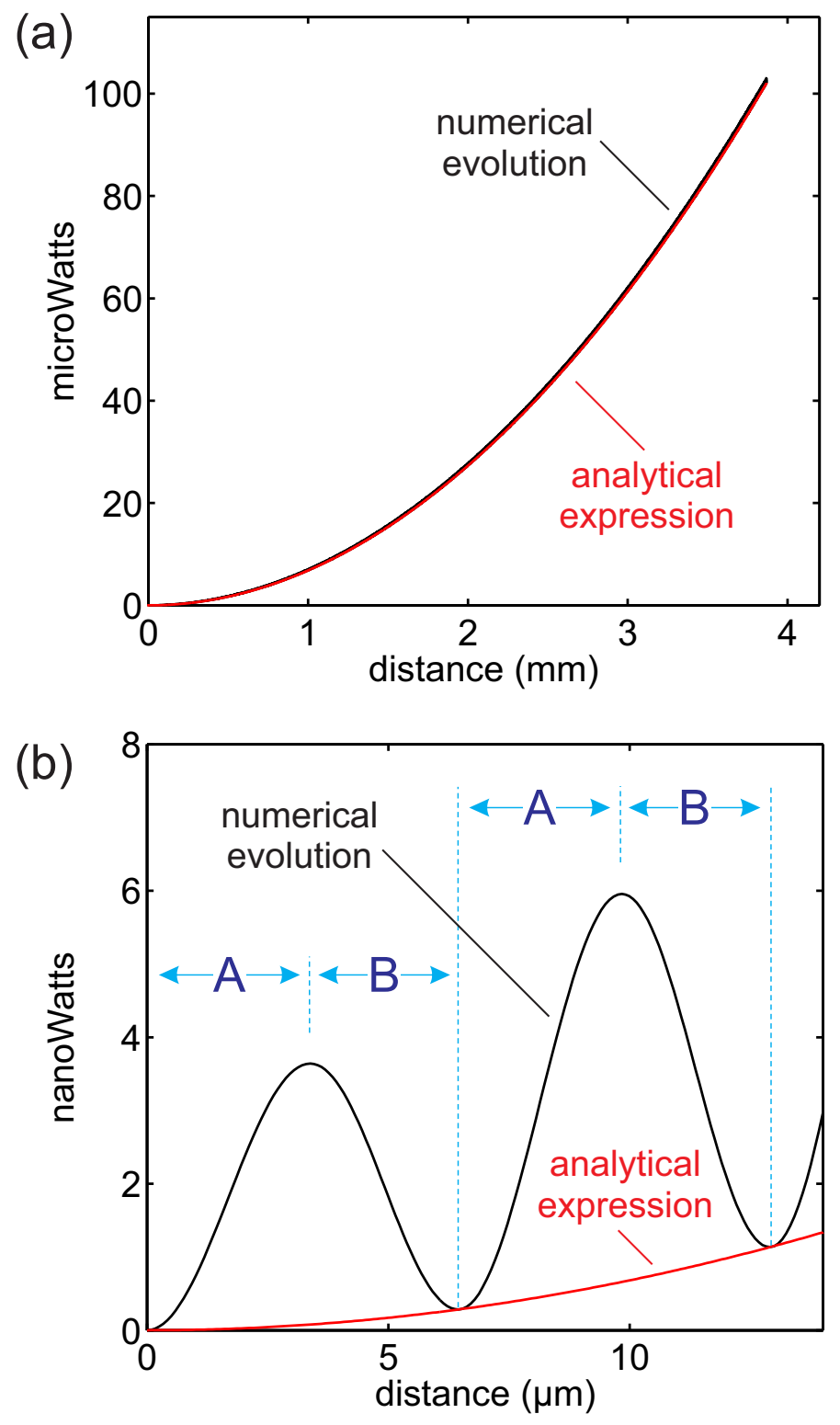

Figure 3: Growth of the difference-frequency field at a wavelength of $3.255 \mu \mathrm{m}$, generated by input waves (100 mW power each) at $1.05 \mu \mathrm{m}$ and $1.55 \mu \mathrm{m}$. The black line is the plot of direct numerical integration of Eq. (4); the red line shows the plot of Eq. (10). 


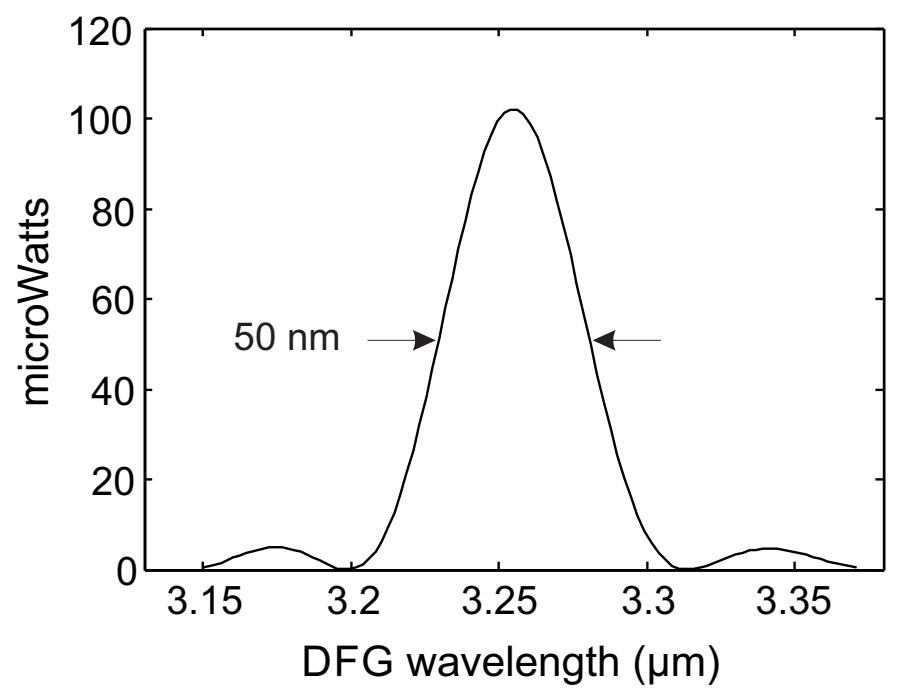

Figure 4: Calculation of generated DFG power versus mid-IR wavelength $\lambda_{1}$, obtained for $\lambda_{2}$ $=1050 \mathrm{~nm}$ and $\lambda_{3}$ ranging from $1525 \mathrm{~nm}$ to $1575 \mathrm{~nm}$, showing a full-width at half-maximum (FWHM) bandwidth of about $50 \mathrm{~nm}$ around $3.255 \mu \mathrm{m}$ for the device discussed in Fig. 2 and the text. 